\title{
CAPÍTULO X \\ PROCESOS DE INNOVACIÓN TECNOLÓGICA EN \\ GRUPOS EMPRESARIALES. EL CASO DE UNA EMPRESA DE AUTOSERVICIOS
}

\author{
Giovanna Patricia Torres Tello \\ hotmail.com

\section{Ruby Asunción González Ascencio} \\ Maestra en Administración. Universidad Autónoma del Carmen \\ Azeneth Cano Alamilla \\ Maestra en Administración, Universidad Autónoma del Carmen
}

Doctora en Estudios Sociales. Universidad Autónoma del Carmen, giovannatt@

\section{Resumen}

El presente estudio tiene el objetivo explicar el proceso de implementación de innovaciones tecnológicas en la empresa de autoservicio Chedraui y su impacto en la organización del trabajo, teniendo como consecuencia el análisis en la toma de decisiones en la implementación de una estrategia empresarial de innovación. Por lo que la implementación de procesos estratégicos de innovación tecnológica, trajo consigo una reorganización del trabajo dentro de la empresa trayendo como efecto la rotación de tareas, la necesidad de un trabajador del tipo polivalente en los autoservicios, nuevos mecanismos de capacitación, de control y vigilancia, así como de procesos distintos de distribución y comercialización. Metodológicamente se orientó bajo el paradigma cualitativo, mediante la revisión documental y bibliográfica para dar soporte teórico al estudio del caso plateado, para la recopilación de información se realizaron 34 entrevistas, de las cuales 19 entrevistados fueron directivos, gerentes de tiendas, jefes de área y trabajadores tanto del corporativo como de los autoservicios. Concluyendo que la implementación de procesos de innovación tecnológica en la empresa, responde a una profesionalización interna en el desarrollo organizacional, dichos procesos son el resultado de una planeación estratégica, direccionada a lograr que los autoservicios se encuentren a la vanguardia de la tecnología en el ramo del autoservicio, así como en los procesos de comercialización y de distribución.

Palabras clave: Innovación, tecnología, grupos empresariales, empresario. 


\title{
PROCESSES OF TECHNOLOGICAL INNOVATION IN BUSINESS GROUPS. THE CASE OF A SELF-SERVICE COMPANY
}

\begin{abstract}
The present study aims to explain the process of implementation of technological innovations in the Chedraui self-service company and its impact on the organization of work, having as a consequence the analysis in decision-making in the implementation of an innovation business strategy. So the implementation of strategic processes of technological innovation, brought about a reorganization of work within the company bringing as an effect the rotation of tasks, the need for a multi-purpose worker in the self-service, new mechanisms of training, control and surveillance, as well as different processes of distribution and marketing. Methodologically oriented under the qualitative paradigm through documentary and bibliographic review to give theoretical support to the study of silver case, for the collection of information 34 interviews were conducted, of which 19 interviewed were managers, store managers, area managers and workers both corporate and self-service. Concluding that the implementation of technological innovation processes in the company, responds to an internal professionalization in organizational development, these processes are the result of strategic planning, aimed at achieving that the self-services are at the forefront of technology in the field self-service as well as marketing and distribution processes.
\end{abstract}

Keywords: Innovation, technology, business groups, businessman.

\section{Capítulo es resultado de un proyecto de investigación culminado}

\section{Introducción}

El desarrollo del presente estudio está centrado en la reconstrucción de las estrategias empresariales que son tomadas como acciones para generar procesos de innovación tecnológica, conduciendo a las organizaciones a unirse en redes y alianzas estratégicas generando grupos empresariales de cooperación mutua, tiene por objetivo explicar el proceso de implementación de innovaciones tecnológicas en la empresa de autoservicio Chedraui y su impacto en la organización del trabajo al interior de la organización, teniendo como consecuencia el análisis en las tomas de decisiones en la implementación de una estrategia empresarial de innovación.

El enfoque con el cual se analizó la implementación de las estrategias empresariales es a partir de que existen diversos factores estructurales y subjetivos, así como diversas redes sociales en diferentes niveles (familia, amistad, compadrazgo, étnico, empresarial, político, empresarial) que las van configurando, partiendo que el empresario es visto como sujeto social en interacción, su subjetividad y acciones le dan sentido a la actividad empresarial, existe en él la búsqueda de la máxima ganancia y de beneficio, además de una racionalidad al hacer negocios y tiene inmerso los procesos de mediación subjetivos y culturales que le dan sentido a una situación concreta. 
El análisis sobre el empresariado permite explicar el éxito o fracaso de una empresa donde éstos pueden configuran diversos códigos que le son utilizados para dar sentido a sus acciones, centrándose en la polémica de si el empresario debe ser concebido como actor racional que siempre se encuentra en la búsqueda de máxima ganancia, teniendo toda la información para calcular sus acciones y obtener soluciones óptimas.

Sin embargo, esta posición o forma de analizar al empresario se percibe un tanto limitada, debido que carece de factores que forman parte de una realidad compleja que permita explicar y comprender las acciones empresariales. Por lo tanto, es imprescindible entender que algunas empresas unidas mediante redes empresariales tienen éxito en el incremento de la innovación, generando resultados positivos, debido a que esos entramados de redes están estrechamente ligados en sistemas de relaciones sociales e industriales, facilitando los procesos de innovaciones tecnológicas debido a sus relaciones e interacción y cooperación mutua.

En tal sentido la investigación se encuentra fundamentada de manera teórica mediante el aporte de diversos autores tal como: Granovetter (2005), Penronse (1955; 1959), Whitley (1994), Bourdieu (1985), Coleman (1990), entre otros. Así como también con el análisis e interpretación de los datos obtenidos de las entrevistas semiestructuradas aplicada a población seleccionada para el estudio

\section{Fundamentación teórica}

\section{Grupos empresariales}

Desde el punto de vista de Granovetter (2005), los grupos empresariales son un conjunto social que jurídicamente son empresas independientes pero unidas en forma persistente, desarrollan diversos tipos de alianzas estratégicas con el fin de maximizar sus ganancias. En este sentido, se entiende a dichos grupos empresariales como redes, o unidades de cooperación, el análisis implica examinarlos desde diferentes ópticas, partiendo de grupos de empresas que poseen relaciones cambiantes con flujos de información asimétricos, es importante destacar que no es posible estudiar estos grupos únicamente desde la visión de los economistas neoclásicos.

Por consiguiente, se consideran los aportes de: Penronse (1955; 1959), Granovetter (2000; 2005) y Whitley (1994), quienes indican que los empresarios o grupos de empresas, vistos como actores, distan mucho de ser simples tomadores de decisión, en función solo de precios y costos para las empresas. Por el contrario, existe una diversidad de arreglos institucionales que influyen en las decisiones y en las diferentes estructuras administrativas y empresariales. De igual manera hace énfasis en la naturaleza contextual de las empresas como agentes económicos y en que los empresarios se mantienen como individuos no aislados en sus actividades empresariales.

Whitley (1994) señala la gran capacidad que poseen los grupos empresariales para crear innovaciones en medios ambientes determinados a partir del establecimiento de redes, ya que existen dos formas de innovación: la "incremental" y la "radical". Innovación "incremental" se entiende por el continuo crecimiento en pequeña escala de productos y procesos de producción, la innovación "radical" son aquellos cambios sustanciales en el producto, ya sea que se introduzcan productos totalmente nuevos o cambios importantes en 
el proceso de producción, en consecuencia, la posibilidad de que se generen las innovaciones depende de que exista en las redes la viabilidad del cambio en los procesos productivos.

Es importante destacar que un grupo puede generar cooperación entre empresarios y se alienta e incentiva a los clientes y proveedores para seguir mejorando los productos o la producción de procesos con la generación de medios ambientes de confianza, solidaridad y reciprocidad en el medio empresarial, esto permite generar una densa red de vínculos empresariales que cuente con la cooperación de las empresas para los intentos de innovación.

Por lo tanto, es imprescindible entender que algunas empresas unidas mediante redes empresariales tienen éxito en el incremento de la innovación, generando resultados positivos, debido a que ese entramado de redes está estrechamente ligadas o embebidas en sistemas de relaciones sociales e industriales, facilitando los procesos de innovaciones tecnológicas debido a sus relaciones e interacción y cooperación mutua, estas prácticas entre empresas según señala Granovette (2000), derivan, de la existencia de una identidad empresarial, factor que determina el comportamiento organizacional y el rendimiento, que es imposible explicar desde un punto de vista puramente económico.

Continuando con los postulados del autor antes mencionado quien contempla algunos ejemplos de la generación de identidad empresarial, basados en los grupos de familias empresariales, con una fuerte dominación que va imponiendo el control de los derechos sobre las empresas, a través de las formas de trabajo en pirámides y la forma de gestión de las empresas para ejercer el control y el poder. No es difícil concluir que algunas familias empresariales, por ejemplo, dominan sus actividades, existiendo filiaciones que se concentran en una sola familia. Este control tiene sentido cuando las familias invierten en la identidad de grupo porque la familia crea un sistema de normas sociales que reduce al intra-grupo a los costos de transacción mediante el fomento de la difusión de la información entre las empresas ${ }^{1}$.

El sentido de identidad empresarial, que Granovetter (2000) señala, permite que las familias empresariales puedan verse amplificadas por otras fuentes de solidaridad. Dicha solidaridad se construye en grupos empresariales, tal es el caso de los grupos étnicos creados a través de la contratación de compatriotas de la misma universidad, la escuela secundaria y la región de origen entre otros. La consecuencia de esta unión es que se refuerza el sentido de la identidad del grupo lo cual facilita la distribución de los recursos. El autor considera que la empresa está sujeta a los ajustes sólo si tiene amplias relaciones con la red empresarial.

Otro rasgo de identidad empresarial, que considera este autor, es el período de duración de un grupo empresarial, pues a mayor duración de un grupo empresarial, mayor identidad en su interior. A esto lo llama acumulación de la tradición. En ocasiones, los objetivos de la familia empresarial dominante pueden entrar en conflicto con la maximización de las empresas filiales que dominan y a veces existen choques financieros en cuento a las familias al momento de redistribuir los recursos en torno a los grupos empresariales, mejorar su condición social aunado a la maximización de los recursos (Granovetter, 2005).

Granovetter en su artículo "Business Groups and Social Organization" (2005) describe a las redes empresariales con formas de prácticas dominantes como el caso de empresas coreanas en donde la empresa matriz limita las estrategias de innovación en cuanto a la financiación, ya que se piensa con este comportamiento puede evitar errores jurídicos. Además, Corea tuvo una centralidad en el estado sobre la producción de las empresas sobre la dominación de la economía, teniendo como resultado una alta centralización de grupos empresariales en donde la forma de negociar se encontraba altamente perneado por relaciones de poder que influyen en las formas de organización empresarial. 
La estructura general de cada país y su estructura social son factores importantes de tomar en cuenta, ya que estos factores se entrecruzan para el desarrollo de grupos empresariales y la evolución de los vínculos de capital humano que poseen diversos canales de información.

Granovetter (2005) menciona que existen cuatro tipos de vínculos empresariales que vigorizan a las redes entre empresas: 1) los préstamos; 2) el comercio; 3) la participación; y 4) la dirección de la expansión empresarial. Estos vínculos se entremezclan en la realidad de los actores y de las instituciones en donde las redes sociales poseen depósitos de identidad que les sirven para la movilización de los recursos, así como para garantizar que los costos de transacción sean bajos a través de las redes empresariales y para producir motivaciones entre los participantes. Pero esta aseveración que hace Granovetter cae deliberadamente en el ámbito de la acción instrumental, porque en ningún momento se habla de la existencia de una configuración entre los distintos niveles de la realidad social del sujeto, es decir, como sujeto que continuamente está reestructurando su realidad por medio de su interacción con los otros y de su percepción del mundo de vida cotidiano.

Además de otros significados basados en los lazos de sangre que generan sentimientos de pertenecía, de confianza, moralidad y compromiso no por la guanacia solamente sino por ser familia. Lo anterior da como consecuencia la influencia de procesos sociales, culturales y subjetivos en la toma de decisiones y la creación de estrategias por parte de los sujetos activos e insertados en densas redes sociales. Y no únicamente existe dentro de las redes la movilización recursos económicos, sino también la existencia de un dinamismo en la movilización de recursos inmateriales como culturales y subjetivos.

Nuestra propuesta en este estudio recae en el análisis de un sujeto empresario visto como sujeto social que genera significados no solo económicos, sino que va estructurando sus acciones dentro de presiones estructurales.

Finalmente, las consideraciones anteriores conducen a mencionar que, dentro del análisis de redes empresariales la cultura, la subjetividad y las acciones de los sujetos activos e incrustados en redes no existen determinismos, sino que existen articulaciones diversas que dependen de la situación a comprender y a analizar. Por lo tanto, existe una relación dialéctica (sujeto/redes) y procesos de decisiones llenos de significaciones de sentido que adquieren, aceptan o rechazan estos sujetos activos en redes sociales.

\section{El capital humano y grupos empresariales}

Bourdieu (1985) señala que los beneficios tanto materiales como simbólicos que reciben los individuos en virtud de la participación en las redes de relaciones sociales y en la construcción de una sociabilidad con el objetivo de crear esos distintos capitales. Bourdieu señala que "las ganancias obtenidas debido a la pertenencia a una red son la base de la solidaridad que las hace posibles" (Bourdieu, 1985). Por lo tanto, el papel de las redes es importante para la generación de capitales distintos (social, cultural), ya que las redes se constituyen mediante estrategias orientadas hacia la institucionalización y socialización de relaciones grupales, utilizables como una fuente confiable de beneficios y de recursos.

Específicamente para Bourdieu (1985), el capital social contiene dos elementos fundamentales: la relación social misma permite a los individuos requerir el acceso a los 
recursos poseídos por la asociación a estas redes y la existencia de la calidad de esos recursos que se obtienen. Es a través del capital social que los sujetos (empresarios) pueden adquirir acceso directo a los recursos materiales (información, conocimientos, mercados protegidos, precios, tecnología, préstamos, etc.) y a su vez puede incrementar el capital cultural de los individuos gracias a la red de relaciones que proporciona contactos con profesionistas o expertos (capital cultural encarnado), o de manera alternativa, la existencia de asociarse a instituciones que otorguen credenciales con valor institucional (capital cultural institucionalizado).

Coleman (1990) define al capital social como "una diversidad de entidades con dos elementos en común: todas consisten en algún aspecto de estructuras sociales que facilitan una cierta acción de los actores - ya se trate de personas (empresarios) o actores corporativos (grandes empresas) - dentro de una estructura". En las empresas, el capital social es una forma de organización social en donde se confía y se coopera para la formación de nuevos grupos y asociaciones. Las prácticas que producen capital social tienden a facilitar la acción que las produce. Granovetter (2000) menciona que algunos de los componentes del capital social son: obligaciones, expectativas y la confianza con lo cual se constituye la creación de redes sociales.

Asimismo, Schiff (1992) define al capital humano como aquel conjunto de elementos acumulados de individuo con una estructura social que interactúa con las relaciones entre las personas, ya que son insumos o argumentos para la producción y/o la función de utilidad (Schiff, 1992). Burt (1992) señala que el capital social son los amigos, colegas y contactos más generales y a través de los cuales el individuo tiene oportunidades de usar su capital financiero y humano.

Algo muy importante que Coleman (1990) había señalado es que la densidad de las redes es una circunstancia importante para el desarrollo del capital social. Mientras tanto, Burt (1992) constató lo contrario a Coleman: es la relativa ausencia de lazos lo que facilita y posibilita la movilidad individual. El argumento anterior se debe a que las redes que son demasiado densas tienden a transmitir información redundante, por lo tanto, los lazos más débiles pueden ser fuentes de nuevos conocimientos y recursos (Granovetter, 2000; Portes, 1998). Portes (1995) señala que el capital social es la capacidad de movilizar recursos de diversos tipos en función de la pertenencia a una red. Por lo tanto, lo que aquí se quiere acentuar es que el empresario realiza sus actividades con relación a la existencia de redes sociales establecidas por los propios sujetos facilitando (o en algunas ocasiones restringiendo) ciertas acciones.

Otro de los enfoques sobre el capital social es el de Putnam (1993) quien lo define como una acumulación de normas y confianza existentes en la red a la cual los individuos recurren para solucionar algunos conflictos que se les presenta en la actividad empresarial. Mientras tanto, Lomnitz (1994) menciona que la reciprocidad que se da entre los miembros de la red se encuentra determinada por el factor confianza, el cual está ligado a elementos culturales (valores, costumbres, etc.), físicos (cercanía de residencia de la empresa) y económicos (intensidad del intercambio empresarial). Por lo tanto, esta autora considera que la confianza es fundamental para llevar a cabo determinadas acciones dentro de las redes sociales y coincidimos en que el capital social en las redes sociales sirve para establecer, adoptar, modificar e intercambiar recursos tanto materiales como inmateriales, así como para la generación de mecanismos de confianza que dan paso a la socialización de relaciones sociales para el beneficio de las redes. 
El capital social en algunos estudios sobre empresas y empresarios tiene que ver con la obtención de recursos e información para el mantenimiento de las empresas en el mercado. Es decir, la relación que existe entre capital social y redes tiene que ver con la creación de conexiones e intercambios instrumentales. Para nosotros, no sólo en las redes se da este intercambio instrumental para crear el máximo beneficio, sino que también se origina intercambios sociales y culturales entre estas estructuras sociales que posibilitan la creación de acciones empresariales.

\section{Procesos de innovación tecnológica en la empresa de autoservicio Chedraui}

En este apartado se explica cómo se dieron los procesos estratégicos de innovación tecnológica en la empresa de autoservicio Chedraui, el modo en el que ocurrió la toma de decisiones y los factores que intervinieron en todo este proceso. El cambio tecnológico que se dio en la empresa comenzó desde 1995 con el ingreso del nuevo director general. Éste comenzó por incorporar tecnología en software para los procesos comerciales, de distribución, así como para la organización de tareas administrativas y gerenciales en las tiendas de autoservicio. Se trató de software con características básicas para el llenado de datos, dado que la recolección de la información se daba muy lentamente.

Así, por ejemplo, los datos financieros de proveedores, de distribución y comercialización tardaban en llegar al corporativo y, por tanto, los estimados de ventas semestrales no se daban con la prontitud necesaria para su procesamiento. Por casi diez años la empresa funcionó con este retraso en sus procesos comerciales, pero es en 2005 que el director general y su equipo de directores toman la decisión de implantar sistemas de procesamiento de la información en sus tres procesos: comercialización, distribución y administración del personal.

El empleo de tecnología de la información tuvo por consecuencia la disminución de costos de operación, verbigracia: inventarios, procesamientos de órdenes de compradistribución de mercancías y reducción de tiempo de las tareas administrativas. Antes de que se aplicara la tecnología de sistemas en 2005, el tiempo de realización de las tareas administrativas se daba aproximadamente en un período de veinte días.

En este sentido, lo que hicieron los directivos del corporativo de tiendas Chedraui fue aprovechar las últimas innovaciones tecnológicas, como fue el caso del Sistema, Aplicaciones y Procesos SAP, haciendo posible un control administrativo y organizativo en las tiendas, agilizando las tareas diarias, disminuyendo los costos de operación e inventarios, lo cual era el propósito de los directivos. Por ejemplo, en el proceso administrativo y de capital humano existe con este sistema una vigilancia intensa y continua en el control presupuestal que cada tienda de autoservicios tiene cada año para sus gastos internos, se tiene control y vigilancia en las adquisiciones que puedan no tener sentido o erogaciones no presupuestadas y por lo cual merece disminuciones presupuestales de las tiendas o el despido de los gerentes, se dispone de información en línea de ingresos y egresos de cada tienda con el objetivo de controlar y vigilar los flujos económicos de la compañía.

La implementación de tecnología en los procesos comerciales agilizó las operaciones, reduciendo en general: número de órdenes devueltas, errores en la elaboración de pedidos y el tiempo de recepción de las mercancías, lo cual a su vez condujo al reforzamiento del proceso logístico de mercancías, a los controles del almacenamiento de productos 
en centros de distribución, de la distribución en tiendas, del pago a proveedores y de las órdenes digitales de compra. En el proceso comercial se reestructuró no únicamente en las relaciones comerciales con los proveedores, sino también en los procesos de planeación comercial que abarcan procesos de resurtido, recibo, facturación y planeación comercial a través de planogramas (la distribución del acomodamiento de las mercancías dentro de los autoservicios y la relación hacia los proveedores).

Además, se innovo en la creación de los planogramas-digitales que mide el desempeño de los muebles rentados a los proveedores, permite tener más productividad dentro de las tiendas al relacionarse con el resurtido de anaqueles y así evitar el agotamiento de anaquel, $\mathrm{y}$, sobre todo, busca incrementar la afluencia de clientes en las tiendas. En este sentido, dicha tecnología estandarizó el proceso comercial con los proveedores, en especial con la generación de datos en promociones, gestión de precios, gestión de órdenes de compra, gestión de stocks, exhibición y señalización dentro de tiendas, todas estas tareas se estandarizaron para un mayor control. Este sistema, denominado Intactix, le posibilitó a la empresa hacer planeaciones estratégicas del resurtido de las mercancías en cada tienda.

Las transformaciones del sistema de distribución y de reparto ha generado un replantamiento de relaciones entre empresa y proveedores, dado que anteriormente eran las tiendas las que se sujetaban a las condiciones de compra; ahora sucede lo contrario, en esta relación el subordinado es el fabricante-proveedor de insumos, ya que si éstos quieren vender y seguir en la preferencia del cliente, tienen que reducir los precios de venta en las mercancías a grandes volúmenes, ya que también la competencia entre los fabricantes de productos similares y a precios más bajos que otros se convierte en un factor que aprovechan las cadenas de autoservicio. De acuerdo a lo anterior, la tecnología tuvo como resultado procesos de control en la empresa.

En el proceso logístico también se innovo por parte de la empresa hacia los proveedores ya que simboliza relaciones de poder, control y acatamiento porque si no se cumple ahora con las normas establecidas en la orden de compra y distribución por parte de los fabricantes hacia la empresa, ésta tiene todas las facultades de sancionar ha dicho proveedor. Lo anterior se refuerza con la inspección y vigilancia del personal interno encargado de esta área, así como de las gerencias en tiendas, en el sentido de que se tiene que poner cuidado en los tiempos de recibo y cumplir la forma de entrega de los productos, así como las cantidades para cada tienda. Si el personal encargado de la supervisión de recibos no ejecuta bien el proceso de revisión de las órdenes de compra, cantidad, tiempo y forma de entrega, esto trae consigo pérdidas para la tienda y afectaciones en la productividad; lo que ocasiona a su vez castigos desde el corporativo y suspensión de bonos de productividad para el personal de las 224 sucursales en el país para el $2016^{2}$.

Por tanto, dicha tecnología trastocó el sistema de incentivos hacia un sistema técnico de control, porque con la más mínima equivocación del gerente o jefe de supervisión de procesos de alguna tienda, el software que acumula toda la información de los procesos de los autoservicios, se pueden suprimir los incentivos a los trabajadores y gerentes, porque al estandarizarse el sistema técnico logístico se establecieron tabuladores de desempeño, los

2 Véase en www.chedraui.com 
cuales sirven para medir el grado de eficacia con el objetivo, en parte, de la reducción de costos de distribución, mermas y daños hacia la mercancía.

De esta forma se aplica una administración científica del trabajo tecnologizada en los procesos comerciales y logísticos aplicando tecnología de última generación en el servicio de retail, del que pueden medir tanto los tiempos de entrega de las mercancías en los centros de distribución (CEDIS) y tiendas, como la medición de la calidad de lo entregado, además se cuantifica en tiempo los kilómetros recorridos por unidad de transporte para la entrega; es decir se califica la puntualidad en los tiempos de entrega de productos tanto en tiendas como en CEDIS.

De la misma manera, se aplican mediciones en tiendas, en específico del lapso que tardan los productos para ser expuestos en los stands y el tiempo que tardan las mercancías para ser vendidas, y hasta el tiempo en que la cajera pasa los productos en el lector óptico, el cual sería el punto final de venta, que es cuando el cliente ya escogió los productos para comprarlos, pues como sabemos el cliente es el eslabón final de la cadena de consumo. Pero la adopción de tecnología para la empresa y en especial para los directivos es significativa porque aumenta el control, el poder y vigilancia hacia los procesos comerciales y de distribución con el objetivo de maximizar las ventas, pero con un control tecnologizado para ser competentes frente a las otras cadenas de autoservicio.

Las contradicciones dadas como consecuencia de la implementación de dicha estrategia, se expresan en actitudes por parte de los trabajadores, tales como el incremento del ausentismo, el robo o daño a las mercancías, enojo y disgusto por el aumento de tareas y por la constante vigilancia por parte de la gerencia, entre otros factores que trastocan las relaciones y los procesos de trabajo. Aunado a esta situación, la empresa puso en marcha un sistema de incentivos de mejora en el tiempo de ejecución de las tareas, los reportes al respecto los conjuntó en una base de datos para medir de los resultados y proyectar el crecimiento de las tiendas y el volumen de ventas en conjunto de todo el negocio de autoservicio.

\section{Procesos de innovación tecnológica y contratación del capital humano en la empresa Chedraui}

El proceso de reclutamiento para tiendas se da por medio de anuncios en los principales periódicos de las ciudades. La recepción de documentos de los candidatos se efectúa en la oficina de recursos humanos de la tienda solicitante, los aspirantes responden a un examen psicométrico en donde se miden aspectos como el trabajo en equipo, conocimientos básicos como sumar, restar, dividir, se mide la respuesta a situaciones concretas que pasarían en tiendas. A los seleccionados se les capacita enseñándoles la estructuración de la empresa, su historia, las percepciones y prestaciones laborales.

En cuanto a capacitación, la empresa implementó un proyecto estratégico imitado de las otras cadenas de autoservicio ${ }^{3}$ consistente en la capacitación vía internet de puestos de las diversas áreas de la tienda, dichos cursos refuerzan la idea de crear parrillas de reemplazo

3 En Wal-Mart existe la Universidad Wal-Mart donde se oferta el bachillerato, licenciaturas relacionadas con el negocio, maestría e inglés. Soriana también tiene su universidad dando también diversos cursos en los niveles de primaria, secundaria, preparatoria, licenciatura y maestría. 
además de un trabajador polivalente. La finalidad es tener asegurado el personal que pueda cubrir las ausencias de los compañeros. El proyecto denominado como Universidad Chedraui, no es otra cosa más que cursos virtuales que se solicitan al jefe de recursos humanos para destinarse a los trabajadores. En el caso del reclutamiento de los jefes de áreas y gerentes se les aplican también exámenes psicométricos como a los trabajadores, que miden ciertas habilidades mentales y de solución de situaciones concretas como saber trabajar en equipo.

La decisión de crear la Universidad Chedraui también fue tomada por los miembros de la familia dueña de la empresa, debido a que la competencia también se encontraba certificando a sus empleados y volviéndolos polivalentes. En 1987, al mando del fundador de los autoservicios, se puso en marcha el Centro de Capacitación Familia (CCF) que instruía a los trabajadores, pero de forma especializada en un sólo oficio, como panadería, cobro en cajas, electrónica, contaduría, por lo que, con el director, la capacitación ahora se reorienta hacia una visión del trabajador polivalente preparado para los reemplazos.

Ahora los trabajadores que aspiren a jefaturas de áreas dentro de tiendas y que se encuentren inscritos en el nuevo Programa de Parrilla de Reemplazo, son exclusivamente para aquéllos que han cubierto un cierto número de cursos de la Universidad Chedraui y cuentan con experiencia en un área determinada en la cual desean ascender. Con la implementación de nueva tecnología en recursos humanos, la empresa ha encontrado ventajas al instaurar el Programa de Parrilla de Reemplazo, particularmente en el caso de la preparación de los trabajadores en el manejo de nueva tecnología como el SAP o el proyecto llamado Crystal, que es la unava tecnología blanda que se implementó en el 2005 en la empresa y la cual modificó el proceso de comercialización y distribución. Lo que les enseña la Universidad Chedraui a los operarios de esta tecnología es la administración de las tareas como los llenados de reportes e informes virtuales en tiempo real.

Por lo que la aplicación de nueva tecnología utilizada tanto para la contratación como para la capacitación de los trabajadores en la empresa. Es un claro ejemplo de que el entorno de competencia en el mercado de los autoservicios en el país determina o presiona a la implementación de nuevas tomas de decisiones en la implementación de tecnología.

\section{Metodología}

El proceso metodológico que oriento esta investigación, se basó en el paradigma cualitativo utilizando la revisión documental y bibliográfica para dar soporte teórico al estudio de caso plateado, que tiene como propósito explicar el procesos de innovación tecnológica implementado en la empresa Chedraui, para lo cual también se aplicaron entrevistas semiestructuradas, permitiendo realizar 34 entrevistas, de las cuales 19 entrevistados fueron directivos, gerentes de tiendas, jefes de área y trabajadores tanto del corporativo como de los autoservicios. La información que se obtuvo de todo el trabajo de campo se dividió en dos niveles de análisis: primer nivel se identificó la estructura organizacional de la empresa, se realizaron entrevistas semiestructuradas y encuestas a mandos medios para conocer sus principales estrategias. En el segundo nivel se identificaron las redes de negocios de las cuales se encontraban vinculadas a los autoservicios, se aplicaron cuestionarios a los empresarios, proveedores y otros sujetos empresariales de los cuales estuvieron dentro de la cadena de valor de la organización. 
Además, se realizó una exhaustiva revisión de documentos como informes de la empresa de autoservicio, manuales de procedimiento, tomas de decisión en la implementación de tecnología, procesos de capacitación, informes sobre estrategias de expansión, distribución, logística y mercadotecnia de los últimos veinte años.

\section{Resultados}

Luego de realizar el recorrido investigativo sobre los procesos de innovación tecnológica en los grupos empresariales tomando como propósito especifico explicar el proceso de implementación de innovaciones tecnológicas en la empresa de autoservicio Chedraui, se identifica que el empresario se encuentra situado en una estructura productiva en la cual también se halla presionado por diferentes factores económicos y del mercado en diversos niveles, que influyen en sus decisiones y que pueden coincidir en intereses, principalmente los que tienen relación con la empresa como el de maximizar las ventas, ganar mercado, implementar tecnología para agilizar los procesos de comercialización y distribución, y así poder tener mayor rentabilidad. Es así que mencionamos a un sujeto empresarial que tiene inmersa una ideología en intereses compartidos que implica una forma de dar sentido y significado a su mundo empresarial.

Las estrategias para lograr los avances en los procesos de innovación tecnológica implementadas por la empresa Chedraui son el resultado de una planeación estratégica dada por la profesionalización interna, en donde el objetivo es que las tiendas Chedraui estén a la vanguardia de la tecnología en el ramo del autoservicio y en sus procesos de comercialización y de distribución, por consiguiente el factor de la competitividad siempre se encuentra presente en las tomas de decisiones, En donde la exploración de la innovación forma parte de las estrategias del actual director general.

Por consiguiente se evidencia los avances del procesos de innovación tecnológica en la empresa Chedraui debido a la la implementación de nueva tecnología en recursos humanos, la empresa ha encontrado ventajas al instaurar el Programa de Parrilla de Reemplazo, particularmente en el caso de la preparación de los trabajadores en el manejo de nueva tecnología como el SAP o el proyecto llamado Crystal, que es la unava tecnología blanda y la cual modificó el proceso de comercialización y distribución, demostrando que apuesta en marcha de la Universidad Chedraui es fundamental para los avances tecnológicos desarrollo y el crecimiento de la empresa generando ventajas competitivas puesto que enseña a los operarios en la administración de las tareas como los llenados de reportes e informes virtuales en tiempo real.

Por consiguiente, nos encontramos que varias estrategias implementadas por la empresa no sólo han respondido a momentos coyunturales como las crisis económicas, sino también la idea de concretarse en el mercado del autoservicio de forma competitiva.

En tal sentido, la existencia de presiones estructurales que enfrenta el sujeto social empresarial tiene que ver principalmente con el crecimiento y expansión del mercado, así como la búsqueda de nuevas formas organizacionales del trabajo, y la creación de trabajadores polivalentes dentro de las tiendas Chedraui. 
Así mismo, para esta empresa la configuración de acumulación de capital en elemento significativo es el de conservar la participación de los integrantes de la familia en el negocio, lo que indica la preservación de una tradición familiar en el cual fue introyectado por el fundador y socializado en los directivos-dueños, conformándose así una identidad propia del hombre de negocios, lo que muestra esta empresa familiar profesionalizada es que ha preservado ya que la familia se supedita a cualquier valor, donde los directivos-dueños en su configuración reflejan que lo significativo es el valor de la familia como factor que define las actividades empresariales, donde invertir en innovaciones tecnológicas es fundamental.

Planear estrategias a futuro es de vital importancia para la empresa para que permitan los avances innovativos y tecnológicos en función del crecimiento empresarial y de capital humano, donde pensar en las actividades de la empresa forma parte de una nueva visión empresarial, que es concebida desde el sujeto social que se encuentra incrustado en la configuración de redes de negocios y de familia de acumulación de capital.

\section{Conclusión}

El sujeto social que analizamos conto con diferentes formas de hacer proyectos empresariales estando siempre en constante interacción con los sucesos del mercado en el ramo de los autoservicios. Analizamos que para la empresa estudiada la configuración de redes negocios con una visión moderna de llevar los negocios profesionalizada los ha llevado a seguir siendo una empresa familiar pero ahora de acumulación de capital a diferencia de su fundador que solo vislumbraba una visión de negocio localista.

Ahora, vemos que el director general, tiene su mirada dirigida hacia aprovechar las ventajas nacionales, regionales y locales con el objetivo de aprovechar la cultura empresarial regional y local acoplándose para el beneficio de la empresa, actualmente para tomar la decisión de invertir o establecer nuevos proyectos empresariales se considera la estabilidad económica, el gobierno de la región, la cultura laboral de la región y local donde encuentran ubicadas los autoservicios.

Estas son parte de las presiones estructurales con las que se enfrentan los sujetos empresariales y que tienen que ver comúnmente con la competencia, el mercado y sus costos. Estos sujetos sociales se constituyen a partir de su relación con la organización de la empresa por han sido empresas con elementos burocratizados. Sin embargo, la subjetividad de los directores les ha resignificado crear nuevos significados organizacionales en la que vierten sus propias valoraciones y emociones de cómo manejar las relaciones de poder, ideología y cultura empresarial en la empresa y con su cuerpo ejecutivo de directores profesionalizados.

Los miembros de la familia Chedraui ahora han articulado nuevas formas de pensar y sentir en su actuar empresarial y cotidiano donde lo subjetivo y sus códigos permean también en las tomas de decisiones de la empresa, así como de una ideología de la empresa con elementos que la llenan de significados como la eficiencia, el control, la responsabilidad y el apego de normas ya establecidas en la empresa. Por lo que responde a una profesionalización en su desarrollo empresarial y en sus estrategias planeadas a largo plazo. Los procesos estratégicos de implantación de tecnología para la empresa son el resultado de una planeación estratégica dada por la profesionalización interna, en donde el 
objetivo es que los autoservicios se encuentren a la vanguardia de la tecnología en el ramo del autoservicio y en sus procesos de comercialización y de distribución.

El factor de la competitividad es algo que siempre se encuentra presente en las tomas de decisiones de los empresarios, donde la exploración de la innovación forma parte de las estrategias del actual director general, Encontramos que varias estrategias no sólo han respondido a momentos coyunturales como las crisis económicas, sino también de la idea de concretarse en el mercado del autoservicio de forma competitiva. La existencia de varias presiones estructurales que se enfrenta el sujeto empresarial tiene que ver principalmente con el crecimiento y expansión del mercado, así como la búsqueda de nuevas formas organizacionales del trabajo y de capital humano. En donde el invertir en innovaciones tecnológicas, el planear estrategias a futuro en donde el pensar en lo nuevo en las actividades de la empresa forma parte de una nueva visión empresarial que es concebida desde el sujeto empresarial que se encuentra incrustado en una configuración de redes de negocios y de familia de acumulación de capital.

\section{Referencias bibliográficas}

Alhama, R. (2001). "Las nuevas formas de organización del trabajo" en artículo publicado en Gestión Polis. México.

Amin, A. y Robins, K. (1991). "Distritos Industriales y desarrollo regional: límites y posibilidades, en revista Sociología del Trabajo. España: Nueva Época, Siglo XXI.

Becattini, G. (1979). "El distrito industrial marshalliano como concepto socioeconómico" en Pyke, D.; Becattini, G.; Senganberg, W. en Los distritos industriales y las pequeñas empresas. España.

Bourdieu, P. (1985). "The forms of capital" en J.G. Richardson (comp.) Handbook of Theory and Research for the Sociology of Education. Nueva York: Greenwood, 241-259.

Burt, R. (1992). Structural holes: the social structure of competition. Cambridge: Harvard University Press.

Coleman, S. (1990). Foundations of Social Theory. Cambridge: Harvard University Press.

Coleman, S. (1988). "Social Capital in the Creation of Human Capital" en American Journal Of Sociology.

De la Garza, E. (1983). El método del concreto-abstracto-concreto. México: UAM-Iztapalapa.

De la Garza, E. (2001). La formación Socioeconómica Neoliberal. México: Plaza y Valdés.

De la Garza, E. (2006a). "Teorías de las organizaciones, entre el actor racional y el neoinstitucionalismo”. Papeles de población. No. 1, México.

De la Garza, E. (2006b). "Los límites de la restructuración productiva en México". Ponencia presentada en el Seminario Internacional. Balance Laboral de dos gobiernos. México y Brasil, Organizado por la UAM y la Universidad de Campiñas. México. 
Della, M. (2001). "Redes sociales y la creación del capital social”. En revista Trabajo, año 2, núm. 4. México.

Guizar, R. (2013). Desarrollo organizacional principios y aplicaciones. México: Mc Graw Hill.

Granovetter, M. (1985). "Economic Action and Social structure: The problem of Embeddednes". en American Journal of Sociology, No. 91, 3, 481-510.

Granovetter, M. (1995). Getting a job: a study of contacts and careers. Chicago: University Press.

Granovetter, M (2000). “A Theoretical Agenda for Economic Sociology". En The New Economic Sociology: Developments in ann Emerging Field. Mauro F. Guillen, Randal Collins, et al. Nueva York: Fundacion Russell Sage, 35-59.

Granovetter, M. (2005). "Business Groups and Social Organization". En Handbook of Economic Sociology, 2nd Edition, Neil and Richard Swedberg Smelser,eds. New Cork and Princeton: Russell Sage Foundation and Princeton University Press.

Luna, M. y Puga, C. (2007). "Los estudios sobre los empresarios y la política. Recuento histórico, líneas de investigación y perspectivas analíticas" en Los estudios de empresarios y empresas. México: Plaza y Valdés.

Llano, C. (1994). El nuevo empresario en México. México: Fondo de Cultura Económica.

Mintzberg, H. (1991). "La estructura de las Organizaciones”. En El Proceso Estratégico. Conceptos, contextos y casos. México: Prentice Hall, 370-392.

Sisto, E. (2014). Fundamentos de administración. México: Trillas.

Penrose, E. (1955). "Research on The Businnes Firms Limits to The Grow and Size of Firms". En American Economic Review, Vol. 45, No. 2, 531-543.

Penrose, E. (1959). Theory of Growth of the Firm. New York: John Wiley \& Sons.

Polanyi, K. (1957). The Great Transformation. Boston: Beacon Press.

Porter, M. (1990). The Competitive Advange of Nations. Oxford: Oxford Press.

Portes, A. (1995). "Economic Sociology and the Sociology of Immigration: a conceptual overview". En Portes, A. (ed.) The Economic Sociologycal of Immigration. Nueva York: Russel Sage Foundation, 1-41.

Portes, A. (1998). “Capital social: sus orígenes y aplicaciones en la sociología moderna”, s/f.

Ponce, R. (2012). Administración, teoría, proceso, áreas funcionales y estrategias para la competitividad. México: McGraw-Hill.

Torres, G (2014). Estrategias y relaciones laborales en los autoservicios Chedraui. Revista Trabajo. Mexico: UAM.

Terry, G. (2012). Administración, Teoría, Proceso, Áreas Funcionales Y Estrategias Para La Competitividad. México D.F.: McGraw-Hill. 